\title{
Synthesis of Novel Fatty Substituted 4-methyl-2H- Chromen-2-one via Cross Metathesis: Potential Antioxidants and Chemotherapeutic Agents
}

\author{
Vyshnavi Yelchuri ${ }^{1}$, R.B.N. Prasad ${ }^{1}$, Mallampalli S.L. Karuna, ${ }^{1, *}$, Y. Poornachandra ${ }^{2}$ and \\ Chityal Ganesh Kumar ${ }^{2}$ \\ ${ }^{1}$ Centre for Lipid Research, CSIR-Indian Institute of Chemical Technology, Hyderabad 500007 , INDIA \\ ${ }^{2}$ Medicinal Chemistry and Pharmacology Division, CSIR-Indian Institute of Chemical Technology, Tarnaka, Hyderabad-500007, INDIA
}

\begin{abstract}
A series of novel fatty substituted 4-methyl-2H-chromen-2-one (coumarins) were synthesized by employing cross metathesis, a key step in the synthesis. The antioxidant activities of the title compounds were compared with the commercial antioxidants, namely butylated hydroxy toluene (BHT) and $\alpha$ tocopherol, glycosidic and other substituted 4-methyl-2H-chromen-2-ones. Among the different 4-methyl2H-chromen-2-ones, the glycosidic substituted 4-methyl-2H-chromen-2-ones was excellent, while those with aliphatic fatty acid chain and hydroxyl substitutents were good. Among the substituted 4-methyl-2Hchromen-2-ones, glycosidic, hydroxyl and cyano containing 4-methyl-2H-chromen-2-ones exhibited good, while fatty substituted exhibited moderate anticancer activities against the four different cancer cell lines tested, namely DU145 (Prostate carcinoma cancer cell), HepG2 (Hepato cellular carcinoma cancer cell), SKOV3 (Ovarian cancer cell) and MDA-MB 231 (Human breast cancer cell). The study reveals that these substituted coumarins can be potential candidates in a number of food and pharmaceutical formulations.
\end{abstract}

Key words: 4-methyl-2H-chromen-2-one, cross metathesis, fatty acids, anticancer activity, antioxidant activity

\section{Introduction}

Coumarins (known as 1, 2-benzo pyrones or O-hydroxycinnamic acid 8-lactones) constitute a major class of phenolic derivatives found in plants comprising of benzene and $\alpha$-pyrone rings ${ }^{1-3)}$. They are natural substances that are known to show anti-tumour activity in vivo, with the effect due to its metabolites (e.g.: 7-hydroxy coumarin). Owing to their wide range of applications in many fields of everyday life such as pharmaceuticals, cosmetics, perfumery and nutrition $^{4-12)}$, their chemistry is being investigated and many natural and non-natural coumarins are being synthesized in recent years ${ }^{13,14)}$.

Importance of free radicals, especially reactive oxygen species (ROS) in pathogenicity of various diseases ${ }^{15,16)}$ including hepatic and vascular diseases ${ }^{17)}$ has attached greater attention of researchers to explore more and more anti-oxidants. In this context, it was observed that additives of 4-methyl-2H-chromen-2-one possessing dihydroxy, diacetoxy and hydroxy-amino groups in the benzene ring at ortho position exhibited very good antioxidant and radical scavenging properties comparably better than $\alpha$-tocopherols ${ }^{18)}$. Thio and oxo analogues of isopsoralen containing 4-methyl-2H-chromen-2-one with 7-oxo and 7-thio moieties derived from the 8-(allyloxy) -4-methyl-2Hchromen-2-one were found to show enhanced activity than those which have seen general clinical use ${ }^{13,14)}$. Matos and coworkers ${ }^{19)}$ synthesized novel amino/nitro substituted 3-aryl-2H-chromen-2-one with nitro, methyl, methoxy, amino and bromo substituents at various positions on the 3-aryl-2H-chromen-2-one scaffold and some of the derivatives exhibited good antibacterial activity against Gram positive and Gram negative bacteria.

As various coumarin derivatives are known to exhibit photophysical and biological activities ${ }^{20)}$, the interest in the synthesis ${ }^{21,22)}$ of these important ring derivatives continues to increase. Also, the interesting biological activities of coumarins and their derivatives have made these coumarins interesting intermediates in organic synthesis. Several synthetic strategies have been employed for the synthesis of coumarins. They are synthesized via Perkin reaction ${ }^{23)}$, Pechman reaction ${ }^{24)}$ or by Knovengel condensation of salicylaldehyde with melonic acid ${ }^{25,26)}$, melonicesters ${ }^{26,27)}$,

*Correspondence to: Mallampalli S.L. Karuna, Centre for Lipid Research, CSIR- Indian Institute of Chemical Technology, Hyderabad 500 007, INDIA

E-mail: karuna@iict.res.in

Accepted July 14, 2016 (received for review October 1, 2015)

Journal of Oleo Science ISSN 1345-8957 print / ISSN 1347-3352 online

http://www.jstage.jst.go.jp/browse/jos/ http://mc.manusriptcentral.com/jjocs 


\section{Yelchuri, R.B.N. Prasad and M. S.L. Karuna et al.}

cyano acetic esters $^{28)}$ or meldrums acid ${ }^{29)}$. Witting reaction in $\mathrm{N}, \mathrm{N}$-diethyl aniline was also employed for the synthesis of coumarins ${ }^{30-32)}$.

A new strategic route for the synthesis of polycyclic coumarin derivatives by the combination of Claisen rearrangement, ring closing metathesis and Diels-Alder reaction was developed by Shital and co-workers ${ }^{20)}$. Metathesis reaction, across a double bond helps in the expansion of diversity and avoids complex reaction steps. Most of the coumarin derivatives prepared by cross metathesis involved allylic functionalities and employed Grubbs' second generation catalyst $^{20,33)}$. Further, Voigtritter et al employed copper salts to enhance the rate of cross metathesis reactions. They observed an increase in reaction rates and yields when CuI was used along with Grubbs' second generation catalyst. Use of CuI salts not only reduces the rate of reaction but also avoids the use of chlorinated solvents ${ }^{34-37)}$. Further carbohydrate conjugated with proteins or lipids play a crucial role in various cellular processes. Oliver et $a l^{38)}$ employed highly selective cross metathesis reaction to develop a variety of glycolipid moieties which were expected to effect the immune system specially the CD-1 media and T-cell activation.

Fatty acids are also known to play a very important role in the synthesis of a number of oleochemicals with industrial and biological applications ${ }^{39)}$. Also, there exists no reports on the synthesis of glycosidic and fatty acid derivatives of coumarins and hence with this background an attempt was made to synthesize hydroxyl, benzyl, glycosidic and fatty acid derivatives of 7-(allyloxy)-4-methyl-2H-chromen-2-one employing conventional method followed by cross metathesis in presence of $\mathrm{CuI}^{40)}$.

\section{Materials and Methods}

\subsection{Materials}

Resorcinol, allyl bromide, potassium carbonate, ethyl acetoacetate, Cis-oleic acid methyl ester (99\%), acrylonitrile, styrene, acrylic acid, allyl alcohol, erucic acid, sulfuric acid,tricyclohexylphosphine [1,3 bis (2,4,6-trimethylphenyl)-4,5-dihydroimidazol2ylidenebenzylidene ruthenium (IV) dichloride (Grubbs' second generation catalyst) (II), butylated hydroxyl toluene (BHT), $\alpha$-tocopherol and 2, 2-diphenyl-1-picrylhydrazyl (DPPH) radical, solvents, namely dry toluene, hexane, ethyl acetate, dry methanol, dry ethyl ether used in the synthesis were of analytical grade and were purchased from M/s Sigma Aldrich Chemical Pvt. Ltd., St. Louis, USA. Castor oil was purchased from M/s Ramcharan Industries Pvt. Ltd., Hyderabad.

\subsection{General procedure for self metathesis of fatty acids}

Fatty acid (0.83 mmol) and Grubbs' second generation catalyst $(0.03 \mathrm{mmol})$ were taken into a two neck round bottom flask $(100 \mathrm{~mL})$. One neck is fixed with septa and another neck with condenser. Two $\mathrm{N}_{2}$ balloons one with needle arranged to the septa and another to the condenser. $20 \mathrm{~mL}$ of dry toluene is added through the septa with the help of syringe followed by Grubb' s second generation catalyst to the reaction mixture maintaining the temperature at $90^{\circ} \mathrm{C}$ and the reaction was carried out for $4 \mathrm{~h}$. Completion of the reaction was monitored by TLC eluted with hexane: ethyl acetate $(90: 10 \mathrm{v} / \mathrm{v})$. The reaction mixture was extracted with ethylacetate, washed with water. Solvent was evaporated using rotary evaporator and dried under vacuum to obtain the product in $92 \%$ yield.

(Z) -7,12-dihydroxyoctadec-9-enedioic acid $\mathrm{C}_{18} \mathrm{H}_{32} \mathrm{O}_{6}$; FT-IR (neat, $\mathrm{cm}^{-1}$ ) : 2898,1735, 1021,758; ${ }^{1} \mathrm{H}$ NMR ( $\delta / p p m$, $\left.\mathrm{CDCl}_{3}\right): 5.50-5.40(\mathrm{~m}, 1 \mathrm{H}), 3.73-3.65(\mathrm{~m}, 1 \mathrm{H}), 2.63-$ $2.50(\mathrm{~m}, 1 \mathrm{H}), 2.43-2.30(\mathrm{~m}, 2 \mathrm{H}), 2.33-2.21(\mathrm{~m}, 1 \mathrm{H}), 2.11$ $-1.92(\mathrm{~m}, 2 \mathrm{H}), 1.80-1.69(\mathrm{~m}, 1 \mathrm{H}), 1.73-1.56(\mathrm{~m}, 3 \mathrm{H})$, $1.45(\mathrm{~d}, J=4.9 \mathrm{~Hz}, 1 \mathrm{H}), 1.35-1.17(\mathrm{~m}, 2 \mathrm{H}) ;{ }^{13} \mathrm{C} \operatorname{NMR}(\delta /$ ppm, $\left.\mathrm{CDCl}_{3}\right): 174.40,128.23,72.28,37.36,36.73,33.80$, 28.99, 25.64, 24.71; ESI-MS $(m / z): 346.12[\mathrm{M}+\mathrm{H}]^{+}$, HRMS: Calcd. for $\mathrm{C}_{18} \mathrm{H}_{33} \mathrm{O}_{6}[\mathrm{M}+\mathrm{H}]^{+}, 346.09$.

(Z)-octadec-9-enedioic acid $\mathrm{C}_{18} \mathrm{H}_{32} \mathrm{O}_{4}$; FT-IR (neat, $\mathrm{cm}^{-1}$ ); $3151,1731,756 ;{ }^{1} \mathrm{H} \mathrm{NMR}\left(\delta / \mathrm{ppm}, \mathrm{CDCl}_{3}\right): 5.36(\mathrm{~m}, 1 \mathrm{H})$, $2.32(\mathrm{t}, J=5.5 \mathrm{~Hz}, 1 \mathrm{H}), 2.10-2.02(\mathrm{~m}, 1 \mathrm{H}), 1.45(\mathrm{~m}, 1 \mathrm{H})$, $1.39-1.30(\mathrm{~m}, 1 \mathrm{H}), 1.31-1.25(\mathrm{~m}, 1 \mathrm{H}), 1.28(\mathrm{~s}, 2 \mathrm{H}) ;{ }^{13} \mathrm{C}$ $\operatorname{NMR}\left(\delta / \mathrm{ppm}, \mathrm{CDCl}_{3}\right): 174.40,130.83,33.60,29.47,28.41$, 27.30, 24.65; $\operatorname{ESI-MS}(m / z): 312.45[\mathrm{M}]^{+}$, HRMS: Calcd. for $\mathrm{C}_{18} \mathrm{H}_{32} \mathrm{O}_{4}[\mathrm{M}]^{+}, 312.89$.

$(Z)$-octadec-9-ene $\mathrm{C}_{18} \mathrm{H}_{36}$; FT-IR (neat, $\mathrm{cm}^{-1}$ ); 3241, 2284, 945; ${ }^{1} \mathrm{H} \operatorname{NMR}\left(\delta / \mathrm{ppm}, \mathrm{CDCl}_{3}\right): 5.39(\mathrm{~m}, 1 \mathrm{H}), 2.12-$ $2.00(\mathrm{~m}, 2 \mathrm{H}), 1.40-1.30(\mathrm{~m}, 2 \mathrm{H}), 1.34-1.21(\mathrm{~m}, 10 \mathrm{H})$, $0.94-0.85(\mathrm{~m}, 3 \mathrm{H}) ;{ }^{13} \mathrm{C} \mathrm{NMR}\left(\delta / \mathrm{ppm}, \mathrm{CDCl}_{3}\right): 130.83,31.68$, 29.51, 28.92, 28.41, 27.30, 22.48, 14.03; $\operatorname{ESI-MS~}(\mathrm{m} / z)$ : 252.27[M] $]^{+}$, HRMS: Calcd. for $\mathrm{C}_{18} \mathrm{H}_{36}[\mathrm{M}]^{+}, 252.94$.

(Z)-hexacos-13-enedioic acid $\mathrm{C}_{28} \mathrm{H}_{48} \mathrm{O}_{4}$; FT-IR (neat, $\mathrm{cm}^{-1}$ ); 3451, 1733, 956; ${ }^{1} \mathrm{H} \mathrm{NMR}\left(\delta / \mathrm{ppm}, \mathrm{CDCl}_{3}\right): 5.39(\mathrm{~m}, 1 \mathrm{H})$, $2.32(\mathrm{t}, J=8.1 \mathrm{~Hz}, 2 \mathrm{H}), 2.10-2.02(\mathrm{~m}, 2 \mathrm{H}), 1.50-1.35(\mathrm{~m}$, $2 \mathrm{H}), 1.39-1.32(\mathrm{~m}, 1 \mathrm{H}), 1.36-1.26(\mathrm{~m}, 10 \mathrm{H}), 1.29-1.20$ $(\mathrm{m}, 5 \mathrm{H}) ;{ }^{13} \mathrm{C} \mathrm{NMR}\left(\delta / \mathrm{ppm}, \mathrm{CDCl}_{3}\right): 174.40,130.83,33.60$, 29.62, 29.57, 28.41, 27.30, 24.65; ESI-MS $(\mathrm{m} / z): 424.07[\mathrm{M}$ $+\mathrm{H}]^{+}$, HRMS: Calcd. for $\mathrm{C}_{28} \mathrm{H}_{49} \mathrm{O}_{4}[\mathrm{M}+\mathrm{H}]^{+}, 424.09$.

\subsection{Synthesis of 7-Hydroxy-4-methyl-2H-chromen-2-one} (5)

To an ice-cold solution of resorcinol (5.5 g, $0.05 \mathrm{~mol})$ in dioxane, conc. $\mathrm{H}_{2} \mathrm{SO}_{4}(2 \mathrm{~mL})$ was added dorp wise under $25^{\circ} \mathrm{C}$. After the addition of concentrated sulphuric acid, ethyl acetoacetate $(7 \mathrm{~mL})$ was added and the mixture was heated to $60^{\circ} \mathrm{C}$ for $4 \mathrm{~h}$. The mixture was then poured into cold water and the precipitate was filtered and dried under reduced pressure. The resulting mixture was recrystallized from methanol to give white needle like crystals, 7-hydroxy-4-methyl-coumarin with $91 \%$ yield. $\mathrm{C}_{10} \mathrm{H}_{8} \mathrm{O}_{3}$; FT-IR $\left(\right.$ neat, $\mathrm{cm}^{-1}$ ): 3021, 2931, 1731, 1438, 1215, 910, 759; ${ }^{1} \mathrm{H}$ 
$\operatorname{NMR}\left(\delta / p p m, \mathrm{CDCl}_{3}\right): 7.52(\mathrm{~d}, J=7.4 \mathrm{~Hz}, 1 \mathrm{H}), 7.00(\mathrm{~s}, 1 \mathrm{H})$, $6.91(\mathrm{~m}, J=7.5,2.0 \mathrm{~Hz}, 1 \mathrm{H}), 6.80(\mathrm{~d}, J=2.0 \mathrm{~Hz}, 1 \mathrm{H}), 6.24$ $(\mathrm{q}, J=1.0 \mathrm{~Hz}, 1 \mathrm{H}), 2.39(\mathrm{~d}, J=1.0 \mathrm{~Hz}, 3 \mathrm{H}) ;{ }^{13} \mathrm{C} \mathrm{NMR}(\delta /$ ppm, $\left.\mathrm{CDCl}_{3}\right): 170.39,157.92,154.92,153.87,125.40$, $114.79,114.00,112.03,101.29,20.03 ; \operatorname{ESI}-M S(m / z)$ : 177.04 $[\mathrm{M}+\mathrm{H}]^{+}$, HRMS: Calcd. for $\mathrm{C}_{10} \mathrm{H}_{9} \mathrm{O}_{3}[\mathrm{M}+\mathrm{H}]^{+}$, 177.19 .

\subsection{Synthesis of 7-allyloxy-4-methyl-2H-chromen-2-one (6)}

To a solution of 7-hydroxy-4-methyl-2H-chromen-2-one (656 mg, $3.73 \mathrm{mmol})$ in acetone $(35 \mathrm{~mL})$, allyl bromide $(520$ $\mathrm{mg}, 4.29 \mathrm{mmol})$ and solid $\mathrm{K}_{2} \mathrm{CO}_{3}(1290 \mathrm{mg}, 9.33 \mathrm{mmol})$ were added and the mixture was stirred at $60^{\circ} \mathrm{C}$ for $6 \mathrm{~h}$. The solvent was evaporated and the residue was extracted into ethyl acetate $(35 \mathrm{~mL})$. The mixture was passed through anhydrous $\mathrm{Na}_{2} \mathrm{SO}_{4}$, concentrated and dried under reduced pressure which furnished compound 7-allyloxy-4-methyl-2H-chromen-2-one as off-white solid prismatic needles in $81 \%$ yield. $\mathrm{C}_{13} \mathrm{H}_{12} \mathrm{O}_{3}$; FT-IR (neat, $\mathrm{cm}^{-1}$ ); 3451, 1736,

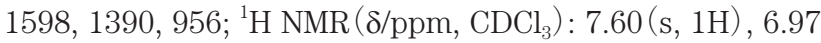
$(\mathrm{s}, 1 \mathrm{H}), 6.92(\mathrm{~s}, 1 \mathrm{H}), 6.24(\mathrm{~d}, J=0.9 \mathrm{~Hz}, 1 \mathrm{H}), 6.05(\mathrm{t}, J=$ $16.5 \mathrm{~Hz}, 1 \mathrm{H}), 5.63-5.54(\mathrm{~m}, 1 \mathrm{H}), 5.39-5.29(\mathrm{~m}, 1 \mathrm{H}), 4.69$ $(\mathrm{s}, 2 \mathrm{H}), 2.39(\mathrm{~s}, 3 \mathrm{H}) ;{ }^{13} \mathrm{C} \mathrm{NMR}\left(\delta / \mathrm{ppm}, \mathrm{CDCl}_{3}\right): 170.39$, $159.94,155.06,153.87,134.52$, 125.35, 117.35, 115.16, $114.20,112.04,101.22,70.02,20.03 ; \operatorname{ESI}-M S(m / z): 217.07$ $[\mathrm{M}+\mathrm{H}]^{+}$. HRMS: Calcd. for $\mathrm{C}_{13} \mathrm{H}_{13} \mathrm{O}_{3}[\mathrm{M}+\mathrm{H}]^{+}, 217.06$.

\subsection{General Procedure for the Synthesis of compound 9a-9f}

A mixture of 7-allyloxy-4-methyl-2H-chromen-2-one (0.50 mmol), compounds 7a-7f (1.50 mmol), Grubbs' second generation catalyst $(8.5 \mathrm{mg}, 10.0 \mu \mathrm{mol})$, and $\mathrm{CuI}$ (2.9 mg, $15.0 \mu \mathrm{mol}$ ) under $\mathrm{N}_{2}$ atmosphere distilled ethyl ether $(5.0 \mathrm{~mL})$ were added, mixture was stirred at $40^{\circ} \mathrm{C}$ for $5 \mathrm{~h}$ in a reflux condenser. After cooling to room temperature, the reaction mixture was concentrated. The solution was heated at $40^{\circ} \mathrm{C}$ for $5 \mathrm{~h}$ followed by cooling to room temperature, then the reaction mixture was passed through anhydrous $\mathrm{Na}_{2} \mathrm{SO}_{4}$ and concentrated under reduced pressure. The crude product was purified by column chromatography and eluted with hexane: ethyl acetate $(60: 40 \mathrm{v} / \mathrm{v})$ to obtain the pure product with $60-78 \%$ isolated yields.

(E) -7-(4-hydroxybut-2-enyloxy)-4-methyl-2H-chromen2-one (9a)

$\mathrm{C}_{14} \mathrm{H}_{14} \mathrm{O}_{4}$; Yield 73.52\%; FT-IR (neat, $\mathrm{cm}^{-1}$ ): 3301, 2918, 2797, 1735, 1504, 716; ${ }^{1} \mathrm{H} \mathrm{NMR}\left(\delta / \mathrm{ppm}, \mathrm{CDCl}_{3}\right): 7.59(\mathrm{~d}, J=$ $7.3 \mathrm{~Hz}, 1 \mathrm{H}), 6.98-6.91(\mathrm{~m}, 2 \mathrm{H}), 6.24(\mathrm{q}, J=1.0 \mathrm{~Hz}, 1 \mathrm{H})$, $5.97-5.85(\mathrm{~m}, 2 \mathrm{H}), 4.71(\mathrm{~m}, 2 \mathrm{H}), 4.20(\mathrm{~m}, 2 \mathrm{H}), 3.68(\mathrm{t}, \mathrm{J}=$ $4.9 \mathrm{~Hz}, 1 \mathrm{H}), 2.39(\mathrm{~d}, J=1.0 \mathrm{~Hz}, 2 \mathrm{H}) ;{ }^{13} \mathrm{C} \mathrm{NMR}(\delta / \mathrm{ppm}$, $\left.\mathrm{CDCl}_{3}\right)$ : 170.39, 159.94, 155.07, 153.88, 133.11, 128.33, 125.35, 115.16, 114.20, 112.04, 101.22, 69.37, 69.37, 63.47, 20.03; $\operatorname{ESI-MS}(m / z): 269.25[\mathrm{M}+\mathrm{Na}]^{+}$, HRMS: Calcd. for
$\mathrm{C}_{14} \mathrm{H}_{14} \mathrm{O}_{4} \mathrm{Na}[\mathrm{M}+\mathrm{Na}]^{+}, 269.32$.

(E) -12-(4-methyl-2-oxo-2H-chromen-7-yloxy) dodec10 -enoic acid (9b)

$\mathrm{C}_{22} \mathrm{H}_{28} \mathrm{O}_{5}$; Yield 68.2\%; FT-IR (neat, $\mathrm{cm}^{-1}$ ): 3151, 2918, 2835, 1725, 1508, 734; ${ }^{1} \mathrm{H} \mathrm{NMR}\left(\delta / p p m, \mathrm{CDCl}_{3}\right): 7.59(\mathrm{~d}, J=$ $7.4 \mathrm{~Hz}, 1 \mathrm{H}), 6.98-6.89(\mathrm{~m}, 1 \mathrm{H}), 6.24(\mathrm{q}, J=1.0 \mathrm{~Hz}, 1 \mathrm{H})$, $5.45-5.66(\mathrm{~m}, 1 \mathrm{H}), 4.71(\mathrm{~m}, 1 \mathrm{H}), 2.39(\mathrm{~d}, J=1.0 \mathrm{~Hz}, 1 \mathrm{H})$, $2.32(\mathrm{t}, J=8.1 \mathrm{~Hz}, 1 \mathrm{H}), 2.11-2.03(\mathrm{~m}, 1 \mathrm{H}), 1.50-1.60(\mathrm{~m}$, $1 \mathrm{H}), 1.39-1.30(\mathrm{~m}, 1 \mathrm{H}), 1.32-1.24(\mathrm{~m}, 1 \mathrm{H}), 1.28(\mathrm{~s}, 3 \mathrm{H})$; ${ }^{13} \mathrm{C} \mathrm{NMR}\left(\delta / \mathrm{ppm}, \mathrm{CDCl}_{3}\right): 174.40,170.38,159.94,155.06$, $153.87,134.17,127.84,125.35,115.16,114.19,112.03$, 101.22, 69.37, 33.61, 31.60, 29.49, 28.41, 25.65, 20.03; $\operatorname{ESI-MS}(m / z): 395.14[\mathrm{M}+\mathrm{Na}]^{+}$, HRMS: Calcd. for $\mathrm{C}_{22} \mathrm{H}_{28} \mathrm{O}_{5}$ $\mathrm{Na}[\mathrm{M}+\mathrm{Na}]^{+}, 395.32$.

(E) -4- (4-methyl-2-oxo-2H-chromen-7-yloxy) but-2-enoic $\operatorname{acid}(9 \mathrm{c})$

$\mathrm{C}_{14} \mathrm{H}_{12} \mathrm{O}_{5}$; Yield 71.8\%; FT-IR (neat, $\mathrm{cm}^{-1}$ ): 3151, 2928, 2857, 1726, 1516, 734; ${ }^{1} \mathrm{H} \mathrm{NMR}\left(\delta / p p m, \mathrm{CDCl}_{3}\right): 7.59(\mathrm{~d}, J=$ $7.4 \mathrm{~Hz}, 1 \mathrm{H}), 7.18(\mathrm{~m}, J=15.2,6.2 \mathrm{~Hz}, 1 \mathrm{H}), 6.99-6.90(\mathrm{~m}$, $2 \mathrm{H}), 6.87(\mathrm{~s}, 1 \mathrm{H}), 6.29(\mathrm{~m}, J=15.0,0.9 \mathrm{~Hz}, 1 \mathrm{H}), 6.24(\mathrm{q}, J$ $=1.0 \mathrm{~Hz}, 1 \mathrm{H}), 4.84(\mathrm{~m}, J=6.1,1.0 \mathrm{~Hz}, 2 \mathrm{H}), 2.39(\mathrm{~d}, J=1.0$ $\mathrm{Hz}, 3 \mathrm{H}) ;{ }^{13} \mathrm{C} \mathrm{NMR}\left(\delta / \mathrm{ppm}, \mathrm{CDCl}_{3}\right): 170.39,169.88,159.94$, 155.06, 153.87, 144.09, 125.35, 120.99, 115.16, 114.19, $112.03,101.22,68.98,20.03 ; \operatorname{ESI}-M S(m / z): 283.14[\mathrm{M}+$ $\mathrm{Na}]^{+}$, HRMS: Calcd. for $\mathrm{C}_{14} \mathrm{H}_{12} \mathrm{O}_{5} \mathrm{Na}[\mathrm{M}+\mathrm{Na}]^{+}, 283.57$.

(E) -4- (4-methyl-2-oxo-2H-chromen-7-yloxy) but-2-enenitrile $(9 \mathrm{~d})$

$\mathrm{C}_{14} \mathrm{H}_{11} \mathrm{NO}_{3}$; Yield 68.2\%; FT-IR (neat, $\mathrm{cm}^{-1}$ ): 3021, 2928, 2757, 1736, 1511, 1220, 756; ${ }^{1} \mathrm{H} \mathrm{NMR}\left(\delta / \mathrm{ppm}, \mathrm{CDCl}_{3}\right): 7.59$ $(\mathrm{d}, J=7.5 \mathrm{~Hz}, 1 \mathrm{H}), 6.98(\mathrm{~m}, J=7.5,2.0 \mathrm{~Hz}, 1 \mathrm{H}), 6.91(\mathrm{~d}, J$ $=2.1 \mathrm{~Hz}, 1 \mathrm{H}), 6.73(\mathrm{~m}, J=15.0,6.2 \mathrm{~Hz}, 1 \mathrm{H}), 6.24(\mathrm{q}, J=1.0$ $\mathrm{Hz}, 1 \mathrm{H}), 5.61(\mathrm{~m}, J=15.0,0.9 \mathrm{~Hz}, 1 \mathrm{H}), 4.71(\mathrm{~m}, J=6.1,1.0$ $\mathrm{Hz}, 2 \mathrm{H}), 2.39(\mathrm{~d}, J=1.0 \mathrm{~Hz}, 2 \mathrm{H}) ;{ }^{13} \mathrm{C} \mathrm{NMR}\left(\delta / \mathrm{ppm}, \mathrm{CDCl}_{3}\right)$ : $170.38,159.94,155.06,153.87,147.78,125.35,118.31$, $115.16,114.20,112.04,101.22,101.00,68.72,20.03$; $\operatorname{ESI-MS}(m / z): 264.24[\mathrm{M}+\mathrm{Na}]^{+}$, HRMS: Calcd. for $\mathrm{C}_{14} \mathrm{H}_{11} \mathrm{NO}_{3} \mathrm{Na}[\mathrm{M}+\mathrm{Na}]^{+}, 264.37$.

(E)-7-(cinnamyloxy)-4-methyl-2H-chromen-2-one (9e)

$\mathrm{C}_{19} \mathrm{H}_{16} \mathrm{O}_{3}$; Yield 76.7\%; FT-IR (neat, $\mathrm{cm}^{-1}$ ): 3051, 2968, 2852, 1738, 1498, 1241, 753; ${ }^{1} \mathrm{H} \mathrm{NMR}\left(\delta / \mathrm{ppm}, \mathrm{CDCl}_{3}\right): 7.59$ $(\mathrm{d}, J=7.5 \mathrm{~Hz}, 1 \mathrm{H}), 7.30$ (m, $J=5.7,4.7,1.7 \mathrm{~Hz}, 2 \mathrm{H}), 7.25-$ $7.16(\mathrm{~m}, 3 \mathrm{H}), 7.01(\mathrm{~m}, J=7.5,2.0 \mathrm{~Hz}, 1 \mathrm{H}), 6.92(\mathrm{~d}, J=2.0$ $\mathrm{Hz}, 1 \mathrm{H}), 6.62(\mathrm{~m}, J=15.2,0.9 \mathrm{~Hz}, 1 \mathrm{H}), 6.36$ (m, $J=15.0,6.2$ $\mathrm{Hz}, 1 \mathrm{H}), 6.24(\mathrm{q}, J=1.0 \mathrm{~Hz}, 1 \mathrm{H}), 4.71(\mathrm{~m}, J=6.1,1.0 \mathrm{~Hz}$, $2 \mathrm{H}), 2.39(\mathrm{~d}, J=1.1 \mathrm{~Hz}, 2 \mathrm{H}) ;{ }^{13} \mathrm{C} \operatorname{NMR}\left(\delta / \mathrm{ppm}, \mathrm{CDCl}_{3}\right)$ : $170.39,159.94,155.07,153.88,135.05,132.28,130.75$, $129.03,127.77,125.35,123.37,115.15,114.20,112.04$, 101.22, 68.72, 20.03; $\operatorname{ESI-MS}(\mathrm{m} / z): 315.32[\mathrm{M}+\mathrm{Na}]^{+}$, HRMS: Calcd. for $\mathrm{C}_{19} \mathrm{H}_{16} \mathrm{O}_{3} \mathrm{Na}[\mathrm{M}+\mathrm{Na}]^{+}, 315.47$.

(E)-2-(acetoxymethyl)-6- (5- (4-methyl-2-oxo-2H-chromen-7-yloxy) pent-3-enyl) tetrahydro-2H-pyran-3,4,5-triyl triacetate $(9 \mathrm{f})$

$\mathrm{C}_{29} \mathrm{H}_{34} \mathrm{O}_{12}$; Yield 67.3\%; FT-IR (neat, $\left.\mathrm{cm}^{-1}\right)$ : 3121, 2738, 2867, 1736, 1508, 1236, $723 ;{ }^{1} \mathrm{H} \mathrm{NMR}\left(\delta / \mathrm{ppm}, \mathrm{CDCl}_{3}\right): 7.59$ 


\section{Yelchuri, R.B.N. Prasad and M. S.L. Karuna et al.}

(d, $J=7.5 \mathrm{~Hz}, 1 \mathrm{H}), 6.98(\mathrm{~m}, J=7.4,1.9 \mathrm{~Hz}, 1 \mathrm{H}), 6.91(\mathrm{~d}, J$ $=2.0 \mathrm{~Hz}, 1 \mathrm{H}), 6.24$ (q, $J=1.0 \mathrm{~Hz}, 1 \mathrm{H}), 5.75-5.65(\mathrm{~m}, 1 \mathrm{H})$, $5.64-5.55(\mathrm{~m}, 1 \mathrm{H}), 5.13(\mathrm{~m}, J=9.5,8.8 \mathrm{~Hz}, 1 \mathrm{H}), 5.07-$ $4.99(\mathrm{~m}, 1 \mathrm{H}), 4.89-4.82(\mathrm{~m}, 1 \mathrm{H}), 4.81-4.73(\mathrm{~m}, 1 \mathrm{H}), 4.64$ (m, $J=12.5,5.7 \mathrm{~Hz}, 1 \mathrm{H}), 4.44(\mathrm{~m}, J=9.3,5.7 \mathrm{~Hz}, 1 \mathrm{H}), 4.05$ $(\mathrm{m}, J=10.9,9.2,1.7 \mathrm{~Hz}, 1 \mathrm{H}), 3.54(\mathrm{~m}, J=12.5,5.7 \mathrm{~Hz}$, $1 \mathrm{H}), 2.39(\mathrm{~d}, J=0.9 \mathrm{~Hz}, 2 \mathrm{H}), 2.25(\mathrm{~m}, 1 \mathrm{H}), 2.11(\mathrm{~s}, 5 \mathrm{H})$, $2.05(\mathrm{~s}, 2 \mathrm{H}), 1.86(\mathrm{~m}, 1 \mathrm{H}), 1.77-1.60(\mathrm{~m}, 1 \mathrm{H}) ;{ }^{13} \mathrm{C} \mathrm{NMR}(\delta /$ ppm, $\left.\mathrm{CDCl}_{3}\right): 170.96,159.94,155.06,153.87,132.20$, $128.94,125.35,115.15,114.20,112.04,101.22,76.36$, 73.23, 71.19, 69.37, 68.60, 67.75, 62.41, 29.47, 28.77, 20.82, 20.57, 20.03; ESI-MS $(m / z): 597.30[\mathrm{M}+\mathrm{Na}]^{+}$, HRMS: Calcd. for $\mathrm{C}_{29} \mathrm{H}_{34} \mathrm{O}_{12} \mathrm{Na}[\mathrm{M}+\mathrm{Na}]^{+}, 597.47$.

\subsection{General Procedure for the Synthesis of compound 10a-10d}

A mixture of 7-allyloxy-4-methyl-2H-chromen-2-one (0.50 mmol),compounds 8a-8d (1.0 mmol), Grubbs' second generation catalyst $(8.5 \mathrm{mg}, 10.0 \mu \mathrm{mol})$, and $\mathrm{CuI}$ (2.9 mg, $15.0 \mu \mathrm{mol}$ ) under an $\mathrm{N}_{2}$ atmosphere distilled ethyl ether $(5.0 \mathrm{~mL})$ were added, mixture was stirred at $40^{\circ} \mathrm{C}$ for $5 \mathrm{~h}$ in a reflux condenser. After cooling to room temperature, the reaction mixture was concentrated under reduced pressure. The crude product was purified by column chromatography and eluted with hexane: ethyl acetate (90:10 v/ v) to obtain the pure product with $60-78 \%$ isolated yields.

(E)-4-methyl-7-(undec-2-enyloxy)-2H-chromen-2-one (10a)

$\mathrm{C}_{21} \mathrm{H}_{28} \mathrm{O}_{3}$; Yield 75.1\%; FT-IR (neat, $\mathrm{cm}^{-1}$ ): 3155, 2857, $1725,1237,726 ;{ }^{1} \mathrm{H} \mathrm{NMR}\left(\delta / \mathrm{ppm}, \mathrm{CDCl}_{3}\right): 6.98-6.89(\mathrm{~m}$, $1 \mathrm{H}), 6.24(\mathrm{q}, J=1.0 \mathrm{~Hz}, 1 \mathrm{H}), 5.77-5.63(\mathrm{~m}, 1 \mathrm{H}), 4.71(\mathrm{~m}, J$ $=5.1,1.0 \mathrm{~Hz}, 1 \mathrm{H}), 2.39(\mathrm{~d}, J=1.1 \mathrm{~Hz}, 1 \mathrm{H}), 2.11-2.03(\mathrm{~m}$, 1H), $1.40-1.28(\mathrm{~m}, 1 \mathrm{H}), 1.31-1.21(\mathrm{~m}, 4 \mathrm{H}), 0.92-0.86$ $(\mathrm{m}, 1 \mathrm{H}) ;{ }^{13} \mathrm{C} \mathrm{NMR}\left(\delta / \mathrm{ppm}, \mathrm{CDCl}_{3}\right): 170.38,159.94,155.06$, $153.88,132.17,129.84,125.35,115.16,114.19,112.03$, 101.22, 69.37, 28.92, 28.41, 22.48, 20.03, 14.03; ESI-MS ( $\mathrm{m} /$ z): $351.20[\mathrm{M}+\mathrm{Na}]^{+}$, HRMS: Calcd. for $\mathrm{C}_{21} \mathrm{H}_{28} \mathrm{O}_{3} \mathrm{Na}[\mathrm{M}+$ $\mathrm{Na}]^{+}, 351.09$.

(E) -11-(4-methyl-2-oxo-2H-chromen-7-yloxy) undec9 -enoic acid (10b)

$\mathrm{C}_{21} \mathrm{H}_{26} \mathrm{O}_{5}$; Yield 78.2\%; FT-IR (neat, $\mathrm{cm}^{-1}$ ): 3211, 2828, 1736, 1508, 1237, 756; ${ }^{1} \mathrm{H} \mathrm{NMR}\left(\delta / \mathrm{ppm}, \mathrm{CDCl}_{3}\right): 7.59(\mathrm{~d}, J=$ $7.5 \mathrm{~Hz}, 1 \mathrm{H}), 6.98(\mathrm{~m}, J=7.5,2.0 \mathrm{~Hz}, 1 \mathrm{H}), 6.91(\mathrm{~d}, J=1.9$ $\mathrm{Hz}, 1 \mathrm{H}), 6.24(\mathrm{q}, J=1.0 \mathrm{~Hz}, 1 \mathrm{H}), 5.76(\mathrm{~m}, 1 \mathrm{H}), 5.67(\mathrm{~m}$, $1 \mathrm{H}), 4.71(\mathrm{~m}, 2 \mathrm{H}), 2.39(\mathrm{~d}, J=1.0 \mathrm{~Hz}, 2 \mathrm{H}), 2.32(\mathrm{t}, J=5.4$ $\mathrm{Hz}, 2 \mathrm{H}), 2.07(\mathrm{~m}, 2 \mathrm{H}), 1.50-1.23(\mathrm{~m}, 5 \mathrm{H}), 1.28(\mathrm{~s}, 3 \mathrm{H}) .{ }^{13} \mathrm{C}$ $\operatorname{NMR}\left(\delta / \mathrm{ppm}, \mathrm{CDCl}_{3}\right): 174.40,170.38,159.94,155.06$, $153.87,132.17,129.84,125.35,115.15,114.19,112.04$, 101.22, 69.37, 33.60, 31.60, 29.47, 28.41, 24.65, 20.03; $\operatorname{ESI-MS}(m / z): 381.17[\mathrm{M}+\mathrm{Na}]^{+}$, HRMS: Calcd. for $\mathrm{C}_{21} \mathrm{H}_{26} \mathrm{O}_{5}$ $\mathrm{Na}[\mathrm{M}+\mathrm{Na}]^{+}, 381.47$.

(E) -12-(4-methyl-2-oxo-2H-chromen-7-yloxy) dodec10-enoic acid (10c)

$\mathrm{C}_{22} \mathrm{H}_{28} \mathrm{O}_{5}$; Yield 69.7\%; FT-IR (neat, $\mathrm{cm}^{-1}$ ): 3051, 2928,

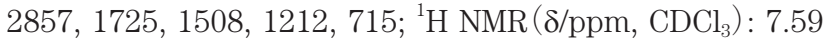
$(\mathrm{m}, J=7.7,1.1 \mathrm{~Hz}, 1 \mathrm{H}), 6.94(\mathrm{~s}, 1 \mathrm{H}), 6.96-6.90(\mathrm{~m}, 1 \mathrm{H})$, $6.24(\mathrm{q}, J=1.0 \mathrm{~Hz}, 1 \mathrm{H}), 5.79-5.62(\mathrm{~m}, 2 \mathrm{H}), 4.74-4.68(\mathrm{~m}$, $2 \mathrm{H}), 2.39(\mathrm{~d}, J=1.0 \mathrm{~Hz}, 2 \mathrm{H}), 2.32(\mathrm{t}, J=8.1 \mathrm{~Hz}, 2 \mathrm{H}), 2.07$ $(\mathrm{m}, 2 \mathrm{H}), 1.50-1.23(\mathrm{~m}, 5 \mathrm{H}), 1.28(\mathrm{~s}, 4 \mathrm{H}) ;{ }^{13} \mathrm{C} \mathrm{NMR}(\delta / \mathrm{ppm}$, $\left.\mathrm{CDCl}_{3}\right): 174.40,170.38,159.94,155.06,153.87,132.17$, $129.84,125.35,115.15,114.20,112.04,101.22,69.37$, 33.60, 31.60, 29.49, 28.41, 24.65, 20.03; ESI-MS $(\mathrm{m} / z)$ : 395.43 $[\mathrm{M}+\mathrm{Na}]^{+}$, HRMS: Calcd. for $\mathrm{C}_{22} \mathrm{H}_{28} \mathrm{O}_{5} \mathrm{Na}[\mathrm{M}+\mathrm{Na}]^{+}$, 395.67 .

(E) -7-(5-hydroxyundec-2-enyloxy) -4-methyl-2H-chromen-2-one (10d)

$\mathrm{C}_{21} \mathrm{H}_{28} \mathrm{O}_{4}$; Yield 75.7\%; FT-IR (neat, $\mathrm{cm}^{-1}$ ): 3251, 2928, 2857, 1736, 1508, 1237, 756; $\left.{ }^{1} \mathrm{H} \mathrm{NMR} \mathrm{( \delta /ppm,} \mathrm{CDCl}_{3}\right) 7.59$ $(\mathrm{d}, J=7.2 \mathrm{~Hz}, 1 \mathrm{H}), 6.96-6.90(\mathrm{~m}, 2 \mathrm{H}), 6.24(\mathrm{q}, J=1.0 \mathrm{~Hz}$, $1 \mathrm{H}), 5.69(\mathrm{~m}, 2 \mathrm{H}), 4.91-4.83(\mathrm{~m}, 1 \mathrm{H}), 4.85-4.77(\mathrm{~m}, 1 \mathrm{H})$, $3.62(\mathrm{~m}, 1 \mathrm{H}), 2.43-2.33(\mathrm{~m}, 4 \mathrm{H}), 2.20-2.11(\mathrm{~m}, 1 \mathrm{H}), 1.91$ $-1.80(\mathrm{~m}, 1 \mathrm{H}), 1.66-1.52(\mathrm{~m}, 2 \mathrm{H}), 1.48-1.07(\mathrm{~m}, 7 \mathrm{H})$, $0.89(\mathrm{t}, J=6.5 \mathrm{~Hz}, 3 \mathrm{H}) ;{ }^{13} \mathrm{C} \mathrm{NMR}\left(\delta / \mathrm{ppm}, \mathrm{CDCl}_{3}\right): 170.38$, $159.94,155.06,153.87,130.15,129.38,125.35,115.16$, $114.19,112.04,101.22,72.28,69.37,40.20,37.36,31.72$, 28.71, 25.64, 22.68, 20.03, 14.03; ESI-MS $(\mathrm{m} / z): 367.44[\mathrm{M}$ $+\mathrm{Na}]^{+}$, HRMS: Calcd. for $\mathrm{C}_{21} \mathrm{H}_{28} \mathrm{O}_{4} \mathrm{Na}[\mathrm{M}+\mathrm{Na}]^{+}, 367.37$.

\subsection{Biological activity}

2.7.1 Antioxidant activity

The antioxidant activity of the synthesized compounds were measured by three in vitro methods such as 2, 2-diphenyl-1-picrylhydrazyl (DPPH) radical activity ${ }^{3,41)}$, superoxide (SO) free radical scavenging activity ${ }^{42)}$ and inhibition of lipid peroxidation ${ }^{43)}$. The $\mathrm{EC}_{50}$ values represent the concentration of the drug at which $50 \%$ of the radicals were scavenged which means the lower $\mathrm{EC}_{50}$ value indicates highest antioxidant activity. For comparison purpose, the $\mathrm{EC}_{50}$ values of commercial antioxidants, namely BHT and $\alpha$-tocopherol were also determined. The tested results are shown in Tables 1 and 2.

2.7.2 Anticancer activity

The synthesisized coumarin derivatives 9a-9f, 10a-10d were further evaluated for in vitro anticancer activity against different cell lines using $\mathrm{MTT}_{\text {assay }}{ }^{44}$ against a panel of tumour cell lines namely DU145, HepG2, SKOV3 and MDA-MB 231.

\section{Results and discussion}

The following steps were involved in the synthesis of a panel of ten novel substituted coumarins. Step 1 is the self metathesis of the substrates such as aliphatic fatty acids and hydroxyl fatty acids prepared from ricinoleic acid, erucic acid and oleic acid(Scheme 1). Step 2 involved Pechman condensation reaction, which involved the synthesis of 7-hydroxy-4-methyl-2H-chromen-2-one by react- 
Substituted coumarins as anti-oxidants and chemptherapeutic agents

Table 1 Antioxidant activity of coumarin derivatives 9a-9f.

\begin{tabular}{crrr}
\hline \multirow{2}{*}{ Compounds } & \multicolumn{3}{c}{$\mathrm{EC}_{50}(\mu \mathrm{g} / \mathrm{mL})$} \\
\cline { 2 - 4 } & DPPH FRSA & $\begin{array}{c}\text { Superoxide } \\
\text { FRSA }\end{array}$ & $\begin{array}{c}\text { Inhibition of lipid } \\
\text { peroxidation }\end{array}$ \\
\hline 9a & $237.1 \pm 0.32$ & $120.0 \pm 0.22$ & $255.3 \pm 0.34$ \\
9b & $17.2 \pm 0.41$ & $17.9 \pm 0.18$ & $18.2 \pm 0.18$ \\
9c & $107.8 \pm 0.29$ & $100.1 \pm 0.36$ & $112.2 \pm 0.26$ \\
9d & $359.5 \pm 0.54$ & $348.3 \pm 0.52$ & $325.3 \pm 0.35$ \\
9e & $48.7 \pm 0.36$ & $32.6 \pm 0.36$ & $43.1 \pm 0.26$ \\
9f & $8.7 \pm 0.26$ & $6.6 \pm 0.16$ & $9.2 \pm 0.21$ \\
BHT & $28.1 \pm 0.21$ & $14.1 \pm 0.22$ & $40.5 \pm 0.22$ \\
$\alpha$-Tocopherol & $10.5 \pm 0.32$ & $7.1 \pm 0.24$ & $20.2 \pm 0.12$ \\
\hline
\end{tabular}

Table 2 Antioxidant activity of coumarin derivatives 10a-10d.

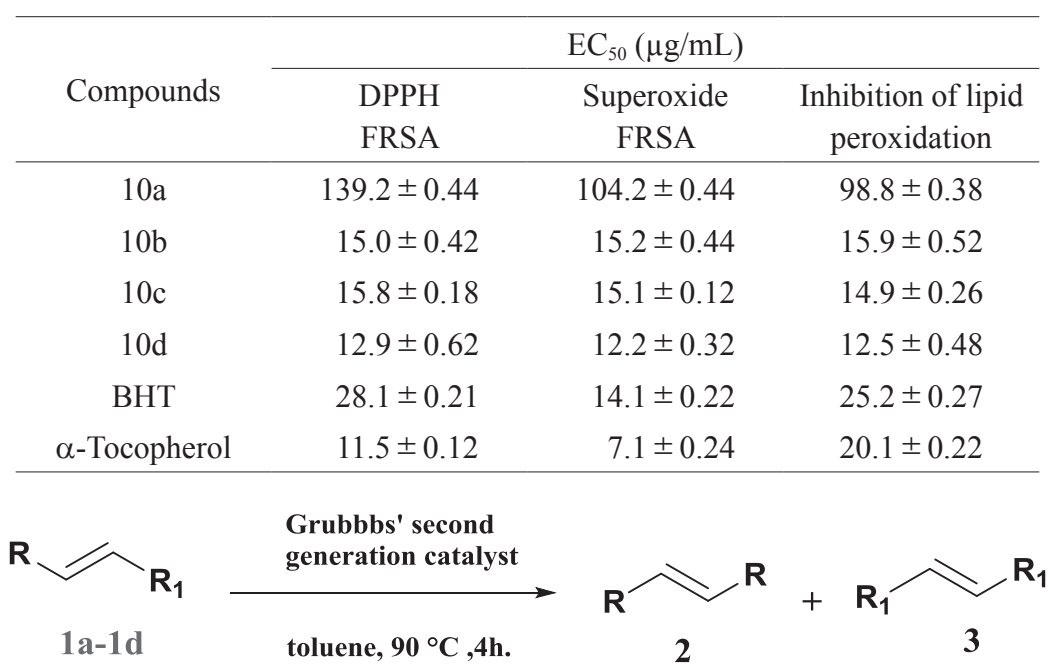
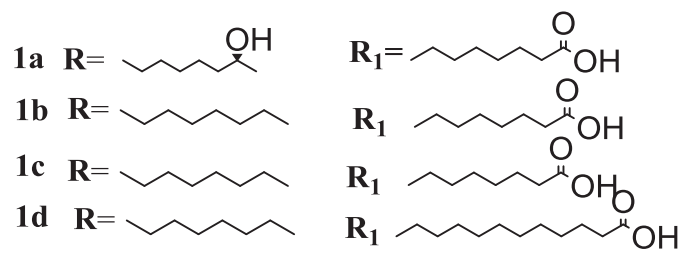

Scheme 1 Self-metathesis of fatty acids.

ing 1,3-dihydroxy benzene with ethyl acetoacetate ${ }^{45)}$ with 95\% yield, in step 3 7-hydroxy-4-methyl-2H-chromen2-one with allyl bromide ${ }^{46)}$ to yield 7-(allyloxy)-4-methyl-2H-chromen-2-one in 91\% yield. While, step 4, a key step in the synthesis is the cross metathesis of 7-(allyloxy) -4-methyl-2H-chromen-2-one with different allylic substitutions such as cyano, hydroxyl, glycosidic and self metathesized fatty acid molecules to yield different coumarin derivatives 9 a-9f and $10 a-10 d$ in $60-78 \%$ yields (Scheme 2 ). With an intention to study the effect of aliphatic fatty acids and aliphatic hydroxyl fatty acids, the double bond in the molecule was coupled to 7-(allyloxy)-4-methyl-2H-chro- men-2-one via cross-metathesis. All the molecules were synthesized employing a simple procedure avoiding multistep reactions in good yields ${ }^{33,47,48)}$ by selecting cross metathesis (Step 4). All the synthesized coumarins were thoroughly characterized using FT-IR, ${ }^{1} \mathrm{H}-\mathrm{NMR},{ }^{13} \mathrm{C}-\mathrm{NMR}$ and mass spectral studies. Coumarin derivatives are known for their good anti-oxidant, anti-cancer and anti-inflammatory behavior $^{49,50)}$. Hence the coumarins synthesized in the present study were evaluated for antioxidant and anticancer properties. 


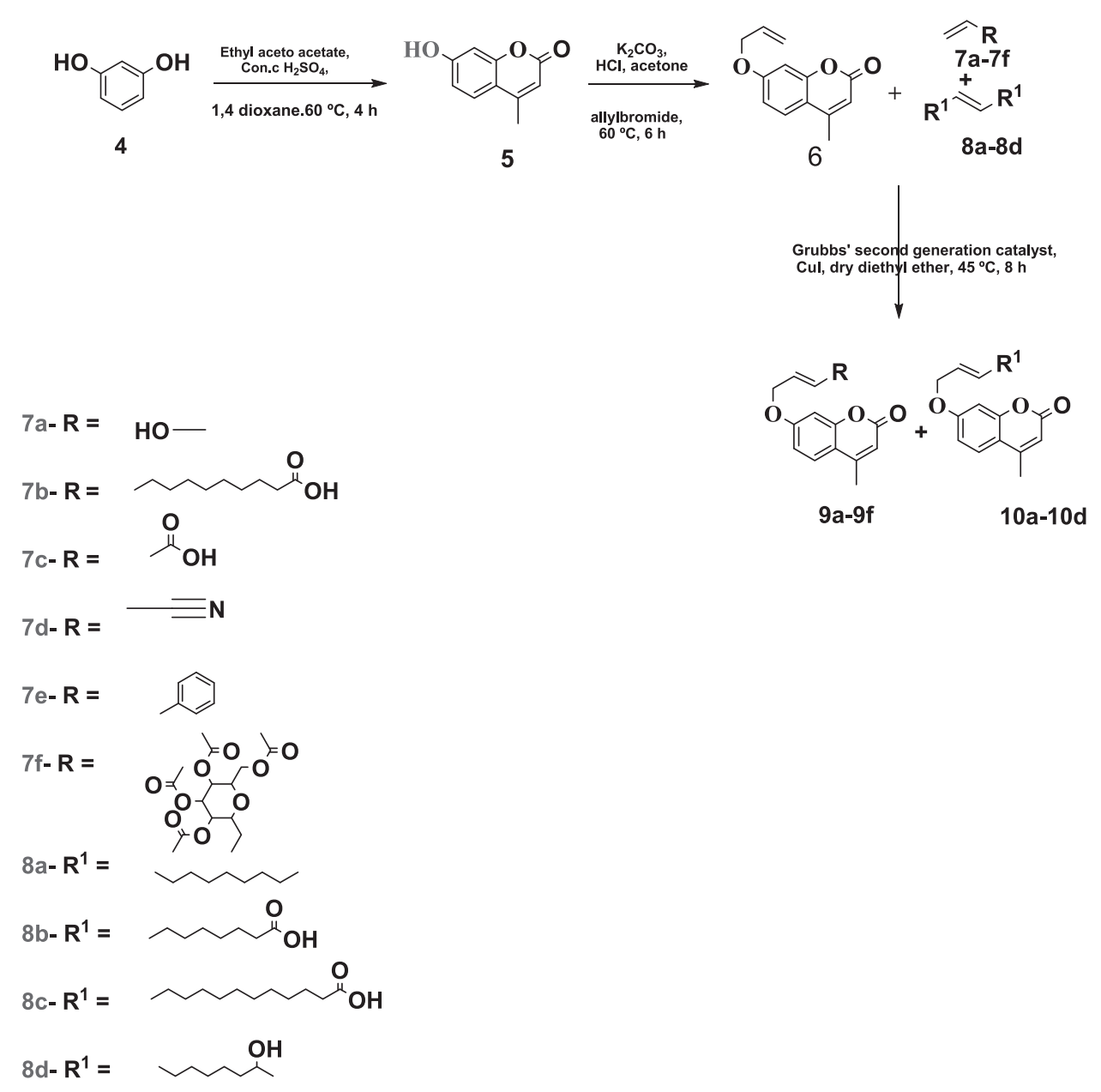

Scheme 2 Synthesis of substituted 4-methyl-2H-chromen-2-one via cross-metathesis.

\subsection{Antioxidant activity}

DPPH radical scavenging method is a simple and widely employed method because of the commercial availability of $\mathrm{DPPH}$ radical. The antioxidant behavior of the coumarin derivatives were compared with the commercial antioxidants, BHT and $\alpha$-tocopherol, as positive controls. It was found that all the compounds exhibited radical scavenging activity in all the three assays performed. Among the different coumarins studied 9a-9f and 10a-10d; 9f, glycosidic derivative of 7-(allyloxy)-4-methyl-2H-chromen-2-one showed the higher antioxidant activity and was better than BHT. The higher antioxidant activity of $9 f$ can be attributed to the fact that the glycosidic molecule coupled to the 4-methyl-2H-chromen-2-one which are themselves active $^{38)}$. This was followed by 9b, 10b, 10c and 10d coumarin derivatives containing aliphatic fatty acid or hydroxyl functionalities. While the rest of the derivatives with hydroxyl, 9a; cyano, 9d and benzyl moieties exhibited poor antioxidant activities. The above coumarin derivatives which exhibited anti-oxidant activity have shown the property even in the absence of native phenolic hydroxyl moiety which could be explained similar to the activities shown by novel coumarin-benzimidazoline derivatives reported by Arora et $a l .{ }^{51)}$.

\subsection{Anti cancer activity}

Among the various substituents coupled to 7-(allyloxy) -4-methyl-2H-chromen-2-one scaffold, the benzyl, 9e; glycosidyl $9 f$ and hydroxyl aliphatic chain containing coumarin, 10d exhibited very good anticancer activities against all tested cell lines. The glycosidic derivatives of 7-(allyloxy)-4-methyl-2H-chromen-2-one exhibited good anticancer activity since the glycolipid is previously known for a number of biological activities ${ }^{52-54)}$. Coumarin derivatives with alkyl and benzyl substituents also known to exhibited good anticancer activity as observed in case of compounds $9 \mathrm{e}$ and $10 \mathrm{~d}$ of the present study ${ }^{55)}$. The fatty substituted coumarin derivatives exhibited moderate activity against the tested cell lines (Table 3). 
Substituted coumarins as anti-oxidants and chemptherapeutic agents

Table 3 Anticancer activity of coumarin derivatives 9a-9f, 10a-10d.

\begin{tabular}{ccccc}
\hline \multirow{2}{*}{ Compounds } & \multicolumn{4}{c}{$\mathrm{IC}_{50}$ values in $(\mu \mathrm{M})$} \\
\cline { 2 - 5 } & DU145 & HepG2 $^{\mathrm{a}}$ & \multicolumn{1}{c}{ SKOV3 $^{\mathrm{c}}$} & MDA-MB 231 $^{\mathrm{d}}$ \\
\hline 9a & $25.3 \pm 0.32$ & $13.6 \pm 0.26$ & $18.2 \pm 0.22$ & $17.7 \pm 0.18$ \\
9b & $46.5 \pm 0.55$ & $44.4 \pm 0.38$ & $39.5 \pm 0.25$ & $31.66 \pm 0.26$ \\
9c & $56.5 \pm 0.55$ & $54.5 \pm 0.38$ & $59.5 \pm 0.25$ & $51.26 \pm 0.25$ \\
9d & $32.8 \pm 0.22$ & $44.5 \pm 0.55$ & $39.5 \pm 0.25$ & $36.6 \pm 0.26$ \\
9e & $12.9 \pm 0.24$ & $13.2 \pm 0.18$ & $12.5 \pm 0.16$ & $10.6 \pm 0.15$ \\
9f & $14.9 \pm 0.24$ & $13.8 \pm 0.18$ & $12.8 \pm 0.16$ & $12.6 \pm 0.25$ \\
10a & $82.9 \pm 0.21$ & $89.2 \pm 0.38$ & $85.1 \pm 0.22$ & $80.5 \pm 0.25$ \\
10b & $78.2 \pm 0.22$ & $85.2 \pm 0.22$ & $77.7 \pm 0.22$ & $79.8 \pm 0.25$ \\
10c & $75.8 \pm 0.22$ & $82.1 \pm 0.22$ & $77.1 \pm 0.22$ & $80.1 \pm 0.25$ \\
10d & $19.0 \pm 0.32$ & $17.3 .5 \pm 0.26$ & $22.6 \pm 0.22$ & $18.3 \pm 0.24$ \\
$\begin{array}{c}\text { Doxorubucin } \\
\text { (control) }\end{array}$ & $0.6 \pm 0.11$ & $0.8 \pm 0.09$ & $0.8 \pm 0.12$ & $0.7 \pm 0.08$ \\
\hline
\end{tabular}

DU145 ${ }^{\mathrm{a}}$ (Prostate carcinoma cancer cell), HepG2 ${ }^{\mathrm{b}}$ (Hepato cellular carcinoma cancer cell), SKOV3 ${ }^{\mathrm{c}}$ (Ovarian cancer cell) and MDA-MB 231 ${ }^{\mathrm{d}}$ (Human breast cancer cell)

\section{Conclusion}

A simple step three procedure was employed for the synthesis of a panel of 10 novel coumarin derivatives. Different molecules such as allyloxy, benzyl, acrylonitrile, acrylic acid and fatty acid analogues were coupled to 4-methyl-2H-chromen-2-one using cross metathesis in step 3 employing Grubb's second generation catalyst in the presence of CuI salt. This is a key step in the synthesis which avoids multi step reactions to obtain the title compounds.

The title compounds exhibited extraordinary to good antioxidant activity against BHT and $\alpha$-tocopherols which are commercially used antioxidants in a number of pharmaceutical formulations. Coumarin derivatives with glycosidic moiety exhibited extraordinary antioxidant activity, while aliphatic fatty acid and alcohol containing derivatives exhibited good antioxidant activities as compared to BHT and $\alpha$ - tocopherol.

The title compounds when evaluated for anticancer activities against four cancer cell lines, namely DU145 (Prostate carcinoma cancer cell), HepG2 (Hepato cellular carcinoma cancer cell), SKOV3 (Ovarian cancer cell) and MDA-MB 231 (Human breast cancer cell) indicated that the glycosidic, hydroxyl and benzyl substituted 4-methyl-2Hchromen-2-ones exhibited good anticancer activities, while other substituted coumarins showed moderate activities, making these candidates potential for use in a number of food and pharmaceutical formulations.

\section{Acknowledgements}

This work was carried out with the financial grant given by Council of Scientific and Industrial Research and was supported by Academy of Scientific \& Innovative Research - Indian Institute of Chemical Technology, India.

\section{Supporting Information}

This material is available free of charge via the Internet at http://dx.doi.org/jos.65.10.5650/jos.ess.15221.

\section{References}

1) Edmont, V.; Stoyanov., Jochen, Mezger., Pechmann reaction promoted by boron trifluoridedihydrate. Molecules 10, 762-766 (2005).

2) Racane, L.; Tralic-Kulenovic, V.; Fiser-Jakic, L.; Boykin, D.W.; Karminski Zamola G., Synthesis of bis-substituted amidinobenzothiazoles as potential anti-hiv agents. Heterocycles 55, 2085-2098(2001).

3) Moon, J.H.; Terao, J. Antioxidant activity of caffeic acid and dihydrocaffeic acid in lard and human lowdensity lipoprotein. J. Agric. Food Chem. 46, 50625065 (1998).

4) Oikawa, T.; Takeuchi, T. Effects of cytogenin, a novel microbial product on embryonic and tumor cell-induced angiogenic responses in vivo. Anticancer Res. 17(3C), 1881-1886 (1997).

5) Patil, A.D.; Freyer, A.J.; Eggleston, D.S.; Haltiwanger, R.C.; Bean, M.F.; Taylor, P.B.; Caranfa, M.J.; Breen, 


\section{Yelchuri, R.B.N. Prasad and M. S.L. Karuna et al.}

A.L.; Bartus, H.R.; Johnson, R.K.; Hertzberg, R.P.; Westley, J.W. The inophyllums novel inhibitors of HIV1 reverse transcriptase isolated from the Malaysian tree Calophylluminophyllum Linn. J. Med. Chem. 36, 4131-4138(1993).

6) Thaisrivongs, S.; Janakiraman, M. N.; Chong, K.T.; Tomich, P.K.; Dolak, L.A.; Turner, S.R.; Strohbach, J.W.; Lynn, J.C.; Horng, M.M.; Hinshaw, R.R.; Watenpaugh, K.D. Structure-based design of novel HIV protease inhibitors: sulfonamide-containing 4-hydroxycoumarins and 4-hydroxy-2-pyrones as potent nonpeptidic inhibitors. J. Med. Chem. 39, 2400-2410 (1996).

7) Hong, H.; Neamati, N.; Wang, S.; Nicklaus, M.M.A.; Pommier, Y.; Burke, T.R.; Zhao, H.; Milne, G.W.A. Discovery of Human Immunodeficiency Virus Type 1 Integrase Inhibitors by Pharmacophore Searching. J. Med. Chem. 40, 930-936 (1997).

8) Murry, R.D.H.; Medez, J.; Brown, S.A. The natural coumarins: occurrence, chemistry and biochemistry. Wiley, New York (1982).

9) Zubia, E.; Luis, F.R.; Massanet, G.M.; Collado, I. An efficient synthesis of furano coumarins. Tetrahedron 48, 4239-4246 (1992).

10) Reich, S.H.; Melnick, M.; Pino, M.J.; Trippe, A.J.; Musick, L. Structure-based design and synthesis of substituted 2-butanols as nonpeptidic inhibitors of HIV protease: secondary amide series. J. Med. Chem. 39, 2781-2794(1996).

11) Michel, A.G.; Durant, F. Methyl 3,4-dihydroisocoumarin-3-carboxylate. Acta Crystallogr. Sect. B 32, 215321 (1976).

12) Schmalle, H.W. ; Jarchow, O.H. ; Hausen, B.M. ; Schulz, K.H. 3,4-Dihydro-8-hydroxy-3- (4-hydroxphenyl) isocoumarin, hydrangenol. Acta Crystallogr. Sect. B 38, 2938-2941 (1982).

13) Rodighiero, P.; Chilin, A. ; Guitto, A. Synthesis of various angular methyl furocoumarins. Gaz. Chim. Ital. 114, 509-515 (1984).

14) Guitto, A.; Chilin, A.; Manzini, P.; Dallacqua, F.; Bordin, F.; Rodighiero, P. Synthesis and antiproliferative activity of furocoumarinisosters. Farmaco. 50, 479-488 (1995).

15) Jakobs, E.A.; Christiaens, L.E.; Rnesen, M. Synthesis of monosulphur and monoselenium analogues of psoralen. Tetrahedron 31, 9315-9324 (1994).

16) Foye, W.O.; Lemke, T.L.; Williams, D.A. Principles of medicinalchemistry fourth ed. BI Wavely Pvt. Ltd. (1995).

17) Heineke, J.W.; Baker, L.; Rosen, H.; Chait, A. Superoxide-mediated modification of low density lipoprotein by arterial smooth muscle cells. J. Clin. Invest. 77, 757-761 (1986).

18) Yogesh, K.; Tyagi; Ajit Kumar.; Hanumantharao, G.;
Raj; Parag Vohra; Garima Gupta; Ranju Kumari; Pankaj Kumar; Rajinder, K.; Gupta. Synthesis of novel amino and acetyl amino-4-methylcoumarins and evaluation of their antioxidant activity. Eur. J. Med. Chem. 40, 413-420 (2005).

19) Matos, M.J.; Vazquez-Rodriguez, S.; Santana, L.; Uriarte, E.; Fuentes-Edfuf, C.; Santos, Y.; Munoz-Crego, A. Synthesis and Structure-Activity Relationships of Novel Amino/Nitro Substituted 3-Arylcoumarins as Antibacterial Agents. Molecules 18, 1394-1404(2013).

20) Shital, K.; Chattopadhyay; Biswas, T., Neogi, K. Synthesis of Polycyclic Coumarin Derivatives by Combined Claisen Rearrangement, Ring-closing Metathesis and Diels-Alder Reaction. Chem. Lett. 35, 376-377 (2006).

21) Murakami, A.; Gao, G.; Omura, M.; Yano, M.; Itlo, C.; Furukawa, H.; Takahasi, D.; Koshimizu, K.; Ohigashi, H. 1,1-Dimethylallylcoumarins Potently Supress both Lipopolysaccharide- and Interferon- induced Nitric Oxide Generation in Mouse Macrophage RAW 264.7 Cells. Bioorg. Med. Chem. Lett. 10, 59-62 (2000).

22) Wu, J.; Liao, Y.; yang, Z. Synthesis of 4 -substituted coumarins via the palladium-catalyzed cross-couplings of 4-tosylcoumarins with terminal acetylenes and organozinc reagents. J. Org. Chem. 66, 3642-3645 (2001).

23) Hepworth, J.D. Comprehensive Heterocyclic Chemistry. Pergamon Press, Oxford 3, 799 (1984).

24) Hepworth, J.D.; Gabbut, C.D.; Heron, B.M. Comprehensive Heterocyclic Chemistry 2nd ed. Pergamon Press, Oxford (1996).

25) Jones, G. Organic Reactions: John Wiley \& Sons: New York 15, 204(1967).

26) Bigi, F.; Chesini, L.; Maggi, R.; Sartori, G. Montmorillonite KSF as an inorganic, water stable, and reusable catalyst for the knoevenagel synthesis of coumarin3-carboxylic acids. J. Org. Chem. 64, 1033-1035 (1999).

27) Knoevenagel, E. Ueber die condensirendeWirkungorganischer Basen. Chem. Ber. 37, 4461-4463 (1904).

28) Wiener, C.; Schroeder, C.H.; Link, K.P. The synthesis of various 3-substituted-4-alkylcoumarins. J. Am. Chem. Soc. 79, 5301-5303(1957).

29) Song, A.; Wang, X.; Lam, K.S. A convenient synthesis of coumarin-3-carboxylic acids via Knoevenagel condensation of Meldrum's acid with ortho-hydroxyaryl aldehydes or ketones. Tetrahedron Lett. 44, 17551758(2003).

30） Ishii, H.; Ishikawa, T.; Wada, H.; Miyazaki, H.; Kanenko, Y.; Harayama, T. Synthetic Studies on Naturally Occurring Coumarins. II. Synthesis of 6, 7-Dimethoxy- and 7, 8-Dimethoxy-5-[(E)-3-oxo-1-butenyl] coumarins. Chem. Pharm. Bull. 40, 2614-2619(1992).

31) Harayama, T.; Nakatsuka, K.; Nishioka, H.; Murakami, 
K.; Hayashida, N.; Ishii, H. Convenient Synthesis of a Simple Coumarin from Salicylaldehyde and Wittig Reagent. II: Synthesis of Bromo- and Methoxycarbonylcoumarins. Chem. Pharm. Bull. 42, 2170-2173 (1994).

32) Harayama, T.; Nakatsuka, K.; Katsuno, K.; Nishioka, H.; Murakami, K.; Fujii, M.; Hayashida, N.; Takeuchi, Y. A Convenient Synthesis of Coumarin Using the Wittig Reagent. Chem. Express 4, 245-248(1993).

33) Arnab, K.; Chatterjee, F.; Dean, Toste; Steven, D.; Goldberg; Robert, H.; Grubbs. Synthesis of coumarins by ring-closing metathesis. Pure Appl. Chem. 75, 421-425 (2003).

34) Lipshutz, B.H.; Aguinaldo, G.T.; Ghorai, S.; Voigtritter, $\mathrm{K}$. Olefin cross-metathesis reactions at room temperature using the nonionic amphiphile "pts" : just add water. Org. Lett. 10, 1325-1328(2008).

35) Lipshutz, B.H.; Ghorai, S.; Leong, W.W.Y. Deprotection of homoallyl (hAllyl) derivatives of phenols, alcohols, acids, and amines. J. Org. Chem. 74, 2854-2857 (2009).

36) Boddaert, T.; Coquerel, Y.; Rodriguez, J. Microwaveassistedcross-metathesis of acrylonitrile. C. R. Chim. 12, 872-875 (2009).

37) Rivard, M.; Blechert, S. Effective and inexpensive acrylonitrile cross-metathesis: utilisation of grubbs ii precatalyst in the presence of copper(i) chloride. Eur. J. Org. Chem. 12, 2225-2228(2003).

38) Plettenburg, O., Mui, C.; Bodmer-Narkevitch, V.; Wong, C.-H. Rapid preparation of glycolipid libraries by cross metathesis. Adv. Synth. Catal. 344, 622-627 (2002).

39) Salimon, J.; Salih, N.; Yousif, E. Industrial development and applications of plant oils and their biobasedoleochemicals. Arabian J. Chem. 5, 135-145 (2012).

40) Voigtritter, K.; Ghorai, S.; Lipshutz, B.H. Rate enhanced olefin cross-metathesis reactions: the copper iodide effect. J. Org. Chem. 76, 4697-4702 (2011).

41) Roussaki, M.; Kontogiorgis, C.; Hadjipavlou-Litina, D.J.; Hamilakis, S. A novel synthesis of 3-aryl coumarins and evaluation of their antioxidant and lipoxygenase inhibitory activity. Bioorg. Med. Chem. Lett. 20, 3889$3892(2010)$.

42) Bors, W.; Heller, W.; Michel, C.; Saran, M. Flavonoids as antioxidants: determination of radical-scavenging efficiencies. Methods Enzymol. 186, 343-355 (1990).

43) Liu, F.; Ooi, V.E.; Chang, S.T. Free radical scavenging activities of mushroom polysaccharide extracts. Life Sci. 60, 763-771 (1997).

44) Zhang, E.X.; Yu, L.J. Studies on polysaccharide from
Sargassumrazilia II for its ability to scavenge active oxygen species. Chinese J. Marine Drugs 3, 1-4 (1997).

45) Xie, S.S.: Wang, X.B.; Jiang-Yan Li, J.Y.; Kong, L.Y. Design, synthesis and biological evaluation of novel 7 -mercaptocoumarin derivatives as $\alpha(1)$-adrenoceptor antagonists. Chem. Pharm. Bull. 61, 16-24(2013).

46) Clarke, D.J.; Robinson, R.S. Synthesis of thio- and oxoanalogues of isopsoralen. Tetrahedron 58, 2831-2837 (2002).

47) Tlenkopatchev, M.A.; Fomine, S.; Fomina, L.; Gavino, R.; Ogawa, T. Ring-opening metathesis polymerization of coumarin-containing norbornene. Polym. J. 29, 622-625 (1997).

48) Schmidt, B.; Krehl, S.; Kelling, A.; Schilde, U. Synthesis of 8-aryl-substituted coumarins based on ring-closing metathesis and suzuki-miyaura coupling: synthesis of a furylcoumarin natural product from galipeapanamensis. J. Org. Chem. 77, 2360-2367 (2012).

49) Riveiro, M.E.; Moglioni, A.; Vazquez, R.; Gomez, N.; Facorro, G.; Piehl, L.; de Celis, E. R.; Shayo, C.; Davio, C. Structural insights into hydroxycoumarin-induced apoptosis in U-937 cells. Bioorg. Med. Chem. 16, 2665-2675 (2008).

50) Belluti, F.; Fontana, G.; Bo, L.; Carenini, N.; Giommarelli, C.; Zunino, F. Design, synthesis and anticancer activities of stilbene-coumarin hybrid compounds: Identification of novel proapoptotic agents. Bioorg. Med. Chem. 18, 3543-3550 (2010).

51) Arora, R.K.; Kaur, N.; Bansal, Y., Bansal, G. Novel coumarin-benzimidazole derivatives as antioxidants and safer anti-inflammatory agents. Acta Pharm. Sin B. 4, 368-375 (2014).

52) Mosmann, T. Rapid colorimetric assay for cellular growth and survival: application to proliferation and cytotoxicity assays. J. Immunol. Methods 56, 55-63 (1983).

53) Liaigre, J.; Dubreuil, D.; Pradere, J.P.; Bouhours, J.F. A novel synthesis of alpha-D-Galp-(1-->3) -beta-D-Galp1-O- (CH2) 3-NH2, its linkage to activated matrices and absorption of anti-alphaGalxenoantibodies by affinity columns. Carbohydr. Res. 325, 265-277 (2000).

54) Bryan, M.C.; Plettenburg, O.; Sears, P.; Rabuka, D.; Wacowich-Sgarbi, S.; Wong, C.H. Saccharide display on microtiter plates. Chem. Biol. 9, 713-720 (2002).

55) Patel, A.K.; Patel, N.H.; Patel, M.A.; Brahmbhatt, D.I. Synthesis characterization and antimicrobial activity of some 4-aryl-2,6-di (coumarin-3-yl) pyridines. ARKIVOC xi, 28-38(2010). 